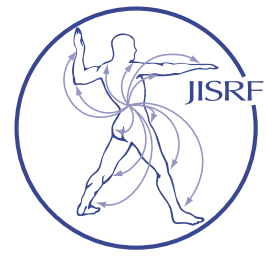

Check for updates

\title{
Elevated Lip Liner Positions Improving Stability in Total Hip Arthroplasty - An Experimental Study
}

Qurashi $S^{1}$, Parr $W^{2}$, Jang $B^{3}$, Walsh $W^{2}$

\section{Abstract}

Background: The use of elevated lip polyethylene liners with the acetabular component is relatively common in Total Hip Arthroplasty (THA). Elevated lip liners increase stability of the THA by increasing the jump distance in one direction. However, the elevated lip, conversely, also reduces the primary arc in the opposite direction and leads to early impingement of the neck on the elevated lip, potentially causing instability.

The aim of the present study is to determine the total range of motion of the femoral head component within the acetabular component with the elevated lip liner in different orientations within the acetabular cup.

Methods: We introduce a novel experimental (ex-vivo) framework for studying the effects lip liner orientation on the range of motion of the femoral component. For constant acetabular cup orientation, the elevated lip liner was positioned superiorly and inferiorly. The femoral component range of motion in the coronal, sagittal and axial plane was measured. To avoid any confounding influences of out of plane motion, the femoral component was constrained to move in the tested plane.

Results: This experimental set up introduces a rigorous framework in which to test the effects of elevated lip liner orientations on the range of motion of the femoral head component in abduction, adduction, flexion, extension and rotation. The movements of this experimental set-up are directly informative of patient's maximum potential postoperative range of motion. Initial results show that an in- ferior placement of the elevated lip increases the effective superior lateral range of motion (abduction) for the femoral component, whilst the anatomy of the patient (i.e. their other leg) prevents the point of femoral component - acetabular lip impingement being reached (in adduction).

\section{Background}

The demands of the patient receiving a modern total hip replacement are ever increasing due to younger and more active patients being operated on. Dislocation continues to be a common complication in total hip arthroplasty (THA). According to the Australian Orthopaedic Association's National Joint Replacement Registry (AOA NJRR), the 14 year cumulative percent revision for primary THA is $9.5 \%$ of which $24.2 \%$ is due to dislocation [2]. Thus, new implant designs, bearing surfaces and the use of muscle sparing surgical approaches claiming increased stability without standard hip precautions are being utilised $[\underline{3}, \underline{4}]$.

Studies on normal physiologic hip Range Of Motion (ROM) have shown varied results with hip flexion and extension ranges of up to 150 degrees, as well as hip abduction and adduction ranges of up to 80 degrees [ㅁ-ㅁ] . It is also accepted that reaching a minimum ROM benchmark is required to achieve a good functional outcome post THA

Keywords: total hip, arthroplasty, dislocation, stability

Level of Evidence: AAOS Therapeutic Level V

Educational Value \& Significance: JISRF Level C 
[]‥ However, this quest for a greater functional ROM in a THA also has to be balanced with stability so as to avoid a dislocation and its consequences. To such an extent, the use of an elevated-rim acetabular liner is widely accepted in THA to improve stability [ㄱ-8]. It was first used by Charnley to decrease posterior dislocations of the femoral head component [9]. Improved stability was first shown by Cobb et al [10] in a retrospective study of elevated-rim liners in THA.

The factors affecting stability from a component position and design perspective are dictated by two key concepts, 'Primary arc' and the 'Jump distance' [11-12]. The total movement of a prosthetic head inside a Polyethylene liner until the point of impingement is known as the 'Primary arc'. The further movement from that point until the point of dislocation is known as the 'Jump distance' (Figure 1).

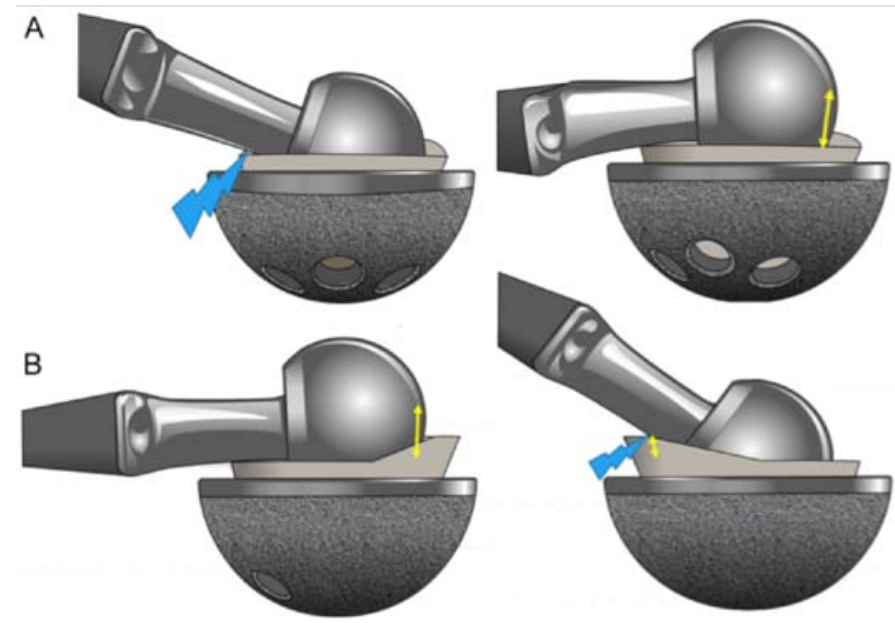

Figure 1 A) Primary arc and jump distance. B) Lip liner increases jump distance in one direction and decreases primary arc in opposite direction.

Any factor that increases the primary arc or jump distance should increase stability $[\underline{11}, \underline{12}]$. Elevated-rim liners improve stability by increasing the jump distance in one direction. However, they have been shown to reduce the primary arc of motion in the opposite direction and lead to impingement (Figure 1b). Impingement between the rim of acetabular component and the neck of the femoral stem is a known cause for dislocation [13]. This occurs by a lever effect of the impingement forcing the femoral head over the acetabular rim, which causes the dislocation. The point of impingement will vary according to the position of the elevated rim in the acetabular shell; impingement will occur more or less in a certain direction depending on the specific plane of movement and the position of the elevated rim.

Shon et al [14] showed in their retrospective retrieval study that the worst combination for impingement, with $92 \%$ prevalence, was the use of an elevated-rim acetabular liner with a femoral neck with extended offset and a flange. They showed that the most common site for impingement was posterior, however, impingement could occur at any location from excessive joint motion. Currently, the postero-superior positioning of the lip liner has been shown to provide additional stability [15], however, a common direction of dislocation is posterior when the hip is flexed and internally rotated $[10,16]$, i.e., posteroinferiorly. Anterior direction of dislocation has also been reported. Yamaguchi et al reported impingement in cases with excessive cup anteversion with posterior positioning of an elevatedrip liner [17].

As well as liner rim positioning, there are several other factors that can increase the incidence of impingement including: acetabular component diameter size; femoral head size; acetabular component positioning and active ROM.

Given the paucity of information in the literature on the effect of elevated-rim liner position and its relation to stability and impingement, the aim of the present study was to investigate impingement points and optimal elevatedrim liner positions. To minimise errors that could be associated with physical testing of ROM in different planes with different rim orientations, we used a validated computational modelling experimental design. Our null hypothesis was that an inferior placement of the lip will increase ROM without any clinically relevant consequent reduction in primary arc in the opposite direction.

\section{Materials and Methods}

A size 1 short offset stem (Profemur L Classic, MicroPort Orthopedics Inc.), 32mm (0) head (Lineage femoral head, MicroPort Orthopedics Inc.), 50mm acetabular component (Dynasty PC Shell, MicroPort Orthopedics Inc.) with a 15 degree lip polyethylene liner (MicroPort Orthopedics Inc., Arlington, TN) were Computer Aided Design (CAD) reverse engineered from the physical parts (tolerance $0.1 \mathrm{~mm}$ ). Collision detection was used to define the impingement limits to the ROM of the femoral stem in the liner part of the CAD model (Figure 2a). The femoral stem was rotated around the centre point of rotation as calculated from the head component $[\underline{18}, \underline{19}]$. The liner orientation was varied and the differences in in-plane ROM recorded.

To validate that the CAD model was accurate in predicting differences in ROM caused by different liner rim positioning, the ROM of the physical construct was measured. This was done by embedding the acetabular cup component in a block of foam so that the femoral component moved along the superior surface of the foam block. This constrains the motion of the femoral component to 


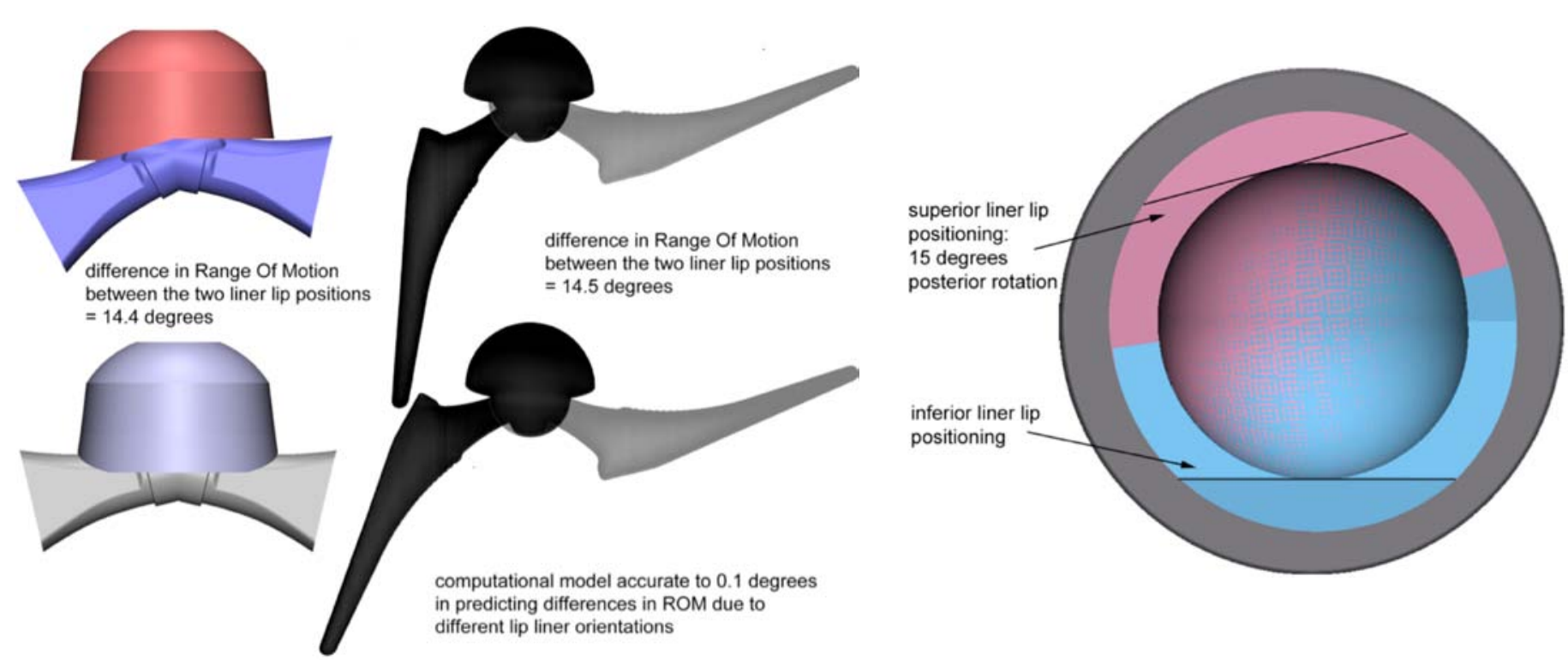

Figure 2a. (Image on left) Difference in range of motion between the two lipped liner positions.

Figure 2b. (Image on right) Positions of lip liner tested in the present study.

Figure 2a. Radiographic validation of the computational femoral stem and liner models. Left hand side shows the computational models of the liner and femoral neck component, right hand side shows (inverted) x-ray images of the femoral component (head and entire femoral stem) and the acetabular component (acetabular cup and liner). The images show that with the difference in range of motion of the femoral component between the two lip liner positions before impinging is 14.5 degrees.

occur within-plane. The set up was mimicked as best as possible in the CAD model (Figure 2a). Due to differences between the CAD and actual model's geometry and set up, the total ROM for the different liner rim positions varied between the CAD and actual parts. The difference in the ROM for the two liner rim positions, which is the focus of the present study, were highly similar (14.4 degrees and 14.5 degrees for the CAD and actual models respectively), validating that the CAD model was suitable for testing the effect of different liner rim positioning on the ROM of the femoral component of a THA.

In Figure $2 \mathrm{a}$, the liner is radio-translucent, therefore not visible in the $\mathrm{x}$-ray images. The top images show the inplane range of motion (ROM) of the femoral component with the lip of the liner orientated to the left (see top left image). The bottom image shows the ROM of the femoral component with the liner lip rotated 90 degrees clockwise compared to the top image. Total in plane ROM of the liner with the lip oriented to the left (top case) for the computational model was 153.4 degrees. For the X-ray model the total ROM was 133.2 degrees. Total in plane ROM of the liner with the lip oriented upwards (bottom case) for the computational model was 139 degrees. For the x-ray model the total range of motion was 118.7 degrees. The difference in the ROM between the computational models was 14.4 degrees. The difference in the ROM between the two $\mathrm{X}$-rays was 14.5 degrees. The computational model is accu- rate to approximately 0.1 degrees in predicting differences in ROM due to different lip liner orientations.

A CT scan of a hip from a 77 year old female was used to create a three-dimensional (3D) isosurface model of the hemipelvis and proximal femur (Figure 3a). The CT DICOM stack was reconstructed using Materialise MIMICS (vs 19.0) software according to methods detailed in Parr et al $[\underline{20}, \underline{21}]$.

The coordinate system for the remainder of the CAD modelling was set according to a 3D isosurface reconstruction of a hip (Figure 3a and Figure 3b). The model was located at $x, y, z=0,0,0$ in the Global Coordinate System (GCS) at the centre point of rotation for the femoral head using Materialise 3Matic software (vs 11.0) (Figure 3a).

For the remainder (the non-validation part) of the CAD experiment the acetabular cup, liner and femoral components were placed in this same coordinate system (Figure $3 b)$.

The acetabular component was positioned with 40 degrees of abduction and 30 degrees of anteversion as this is the ideal acetabular cup position suggested by Scheerlinck [22]. We also acknowledge that there is significant variability in this range and the formerly described Lewinnek 'safe zones' have since been shown to vary based on the dynamics of the patient as well as the pelvis position in the sagittal plane changes throughout different stance positions and functional activities [23, 24]]. 


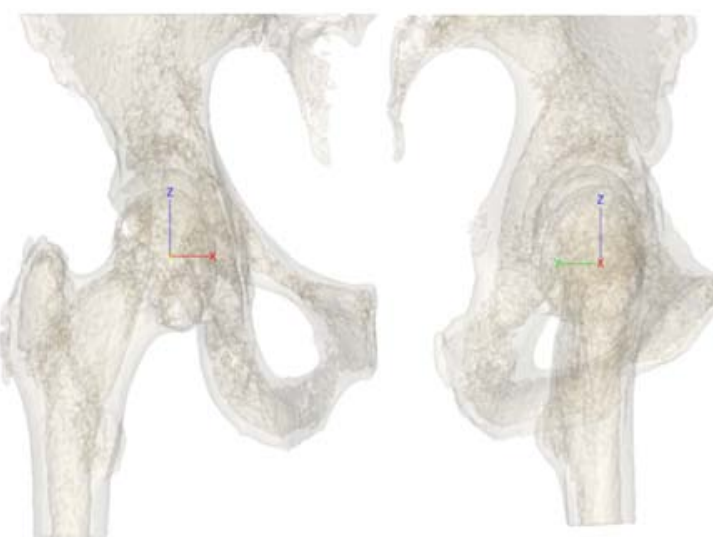

Figure $3 a$

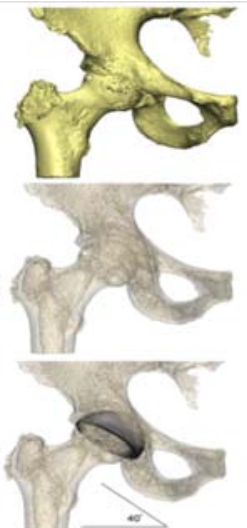

Figure $3 b$

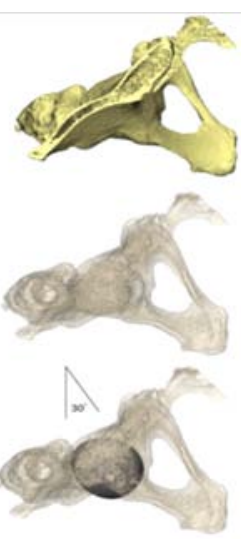

Results

In Table 1, hip flexion, extension and abduction was greater when the elevated lip liner was positioned in the inferior position compared to the superior position. Hip adduction, internal and external rotations were greater when the liner was positioned in the superior position. The results of the combined movements of rotation around the three axes with the stem held in 90 degrees of flexion considering the clinical relevance of this particular movement are presented in Table 2 and illustrated in Figure $4 \mathrm{~b}$.

This shows that an inferiorly

This position as well as the pelvis was fixed throughout the study with the femur rotating about the centre of rotation of the hip joint (x,y,z=0,0,0 in the GCS). The liner within the fixed position acetabular cup was placed in two orientations: a superior orientation with the apex of the elevated rim rotated posteriorly by 15 degrees; and an inferior position (Figure $2 b$ ).

The femoral component of the CAD model was moved about the centre of rotation (COR) of the head component (which was set at the GCS x,y,z $=0,0,0$, see above). The movements of the femoral component were constrained to be planar: moving in the axial, coronal and sagittal planes around the z, y, x axes respectively (see Figure 3a, Figure $4 \mathrm{a}$ ). These planar movements corresponded with the following femoral component movements: sagittal plane movement around the $\mathrm{x}$ axis for flexion and extension; coronal plane movement around the y axis for abduction and adduction; axial plane movement around the $\mathrm{z}$ axis for internal and external rotation.

Total ROM (in degrees) of rotation were recorded from maximum negative rotation and maximum positive rotation around each axis. Minimal and maximal points were determined when femoral neck component impingement (collision) with liner were detected in the CAD models. ROM was measured with the liner lip in two positions, with the elevated lip superior (with 15 degrees of posterior rotation) and inferior (Figure $2 b$ ).

Additionally, one mixed movement scenario was simulated where the femoral component was rotated in the axial plane (around the $\mathrm{z}$ axis) with the femoral component positioned in 90 degrees flexion (rotated anteriorly by 90 degrees around the $\mathrm{x}$ axis). placed lip will allow more than twice the amount of internal rotation in a flexed position when compared with the lip positioned postero- superiorly.

Table 1.

\begin{tabular}{|l|l|}
\hline $\begin{array}{l}\text { Femoral component } \\
\text { direction }\end{array}$ & $\begin{array}{l}\text { Angle difference in degrees (inferior lip } \\
\text { liner compared to superior lip liner) }\end{array}$ \\
\hline Flexion & $17.2^{\circ}$ \\
\hline Extension & $37^{\circ}$ \\
\hline Abduction & $16.8^{\circ}$ \\
\hline Adduction & $-17^{\circ}$ \\
\hline Internal rotation & $-18.6^{\circ}$ \\
\hline External rotation & $-18.2^{\circ}$ \\
\hline
\end{tabular}

Table 2. ROM of femoral stem component for rotation in the coronal plane with the femoral component at 90 o flexion

\begin{tabular}{|l|l|l|}
\hline $\begin{array}{l}\text { Liner Lip } \\
\text { Position }\end{array}$ & $\begin{array}{l}\text { Internal (superior) } \\
\text { rotation in the coronal } \\
\text { plane }\end{array}$ & $\begin{array}{l}\text { External (inferior) } \\
\text { rotation in the coronal } \\
\text { plane }\end{array}$ \\
\hline Superior & $16.8^{\circ}$ & $143.6^{\circ}$ \\
\hline Inferior & $35.2^{\circ}$ & $130.6^{\circ}$ \\
\hline
\end{tabular}

\section{Discussion}

Dislocation continues to be a major complication after total hip arthroplasty [2].The causes of dislocation can be generally ascribed to four factors: soft tissue tension; soft tissue function; component design; component position [르]. These can, in isolation or in combination, result in a dislocation.

Component design and component position are the fac- 


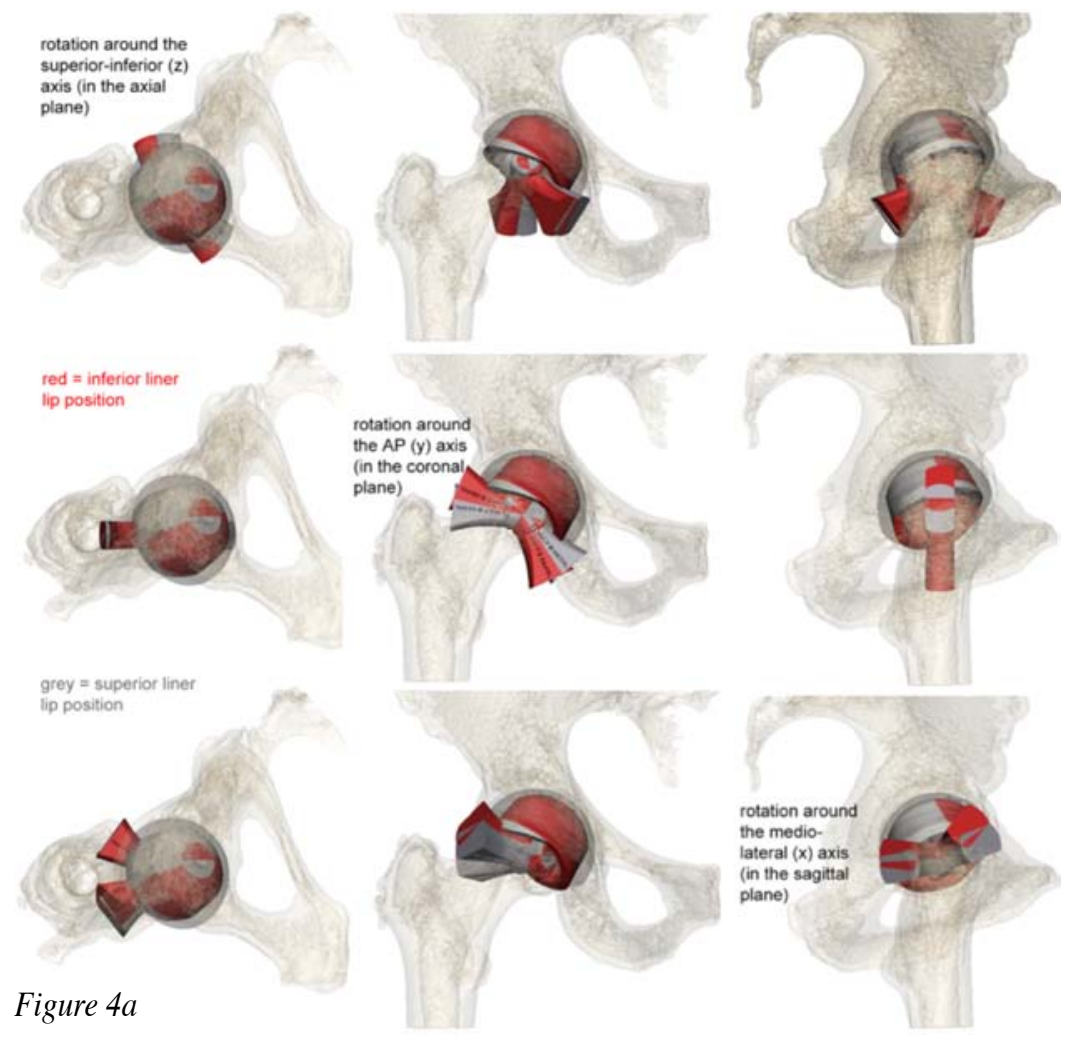

pingement damage shown on retrieval studies $[\underline{14}, \underline{27}, \underline{28}]$. Elevated lip liners were first used by Charnley in the early 1970s to prevent posterior hip dislocation and more recently have been shown to increase stability $[\underline{10}, 2, \underline{26}]$.

Our results show that an inferior placement of the elevated lip liner allows increased effective coronal (abduction) as well as sagittal plane range of motion for the femoral component (Figure 4a, Figure 4b, Table 2, Table 3).

Our study shows that an increase in range in flexion, extension and abduction with an inferiorly placed liner lip but a reduction in rotation and adduction. But is this likely to have a negative effect by increasing impingement? Reduction in ROM due to early impingement is undesirable, however, is the reduction in rotation and adduction of any clinical significance? To answer this question, we need to know what the physiological range of motion should be.

There are various studies [ㅁ-ㅁ] looking at native hip ranges which indicate that the reduction of range in rotation as a result of the extended
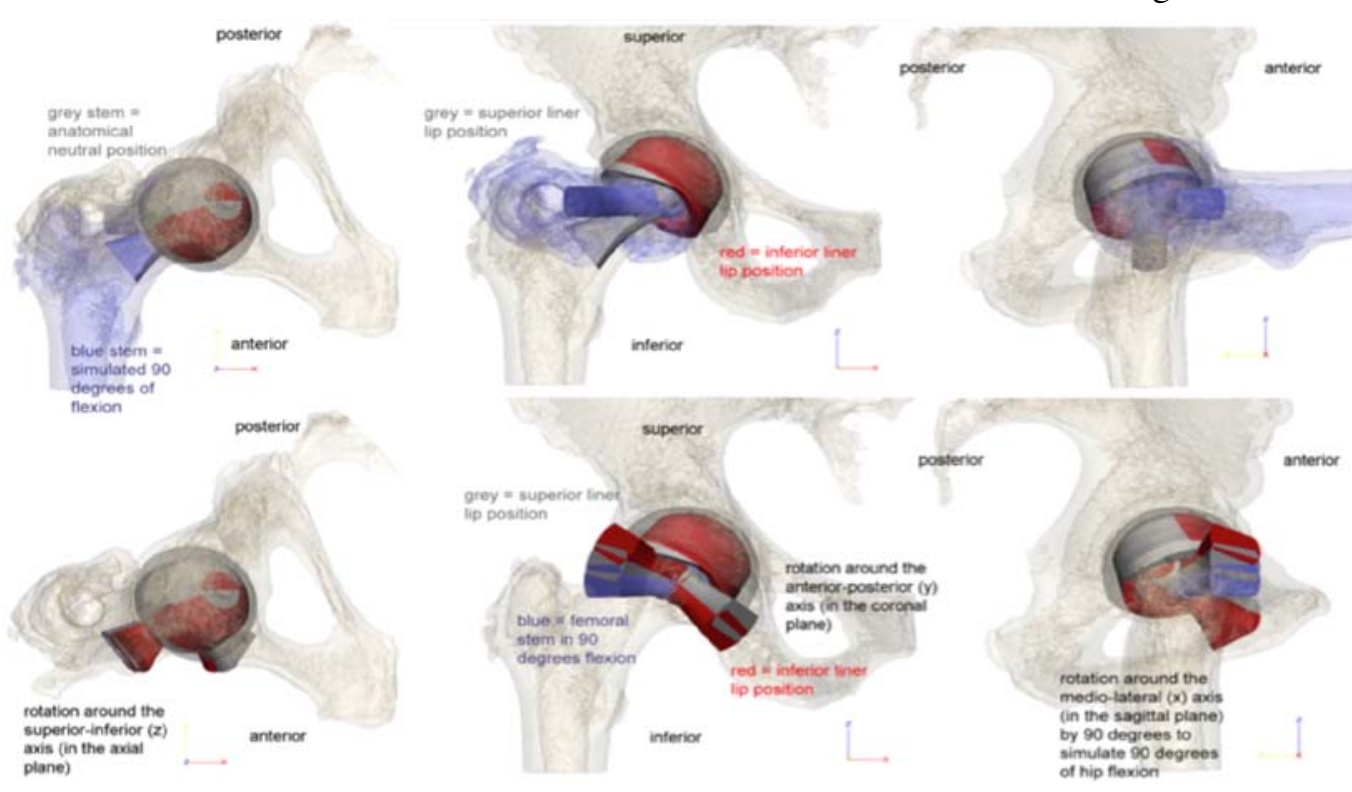

Figure $4 b$

Figure 4a) Assessment of 'primary arc' range with Lip in variable orientations.4b) Images show (left to right) construct in axial, coronal and sagittal planes. Top images show the simulation of the femur and femoral stem component in 90 degrees of flexion. Bottom images show the combination movements in the three planes with the femoral stem starting in 90 degrees of flexion.

tors where mechanical impingement is thought to be the culprit and the earlier discussed concepts of primary arc and jump distance come into play $[\underline{11,26]}$. Most dislocations are thought to occur secondary to mechanical impingement [드] and much literature discusses dislocation secondary to femoral-neck-on-liner impingement with im-

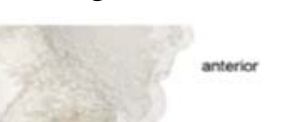

lip being inferiorly placed is not, for the vast majority of the population, an issue. This is because the overall arc of motion in rotation should be approximately 150 degrees []. The loss of motion of 18 degrees (from an inferior lip) will result in a residual arc of over 110 degrees, which is greater than the axial rotational arc in most studies [므므.

Physiological hip rotational studies show limited data on normal hip rotation range of motion in adults. Kouyoumdjian et al. noted rotation in bilateral physiological hips to be symmetrical with predominance for external rotation [29]. Cibulka et al found external rotation to be predominant in 52\% of patients [30]. Widmer et al. defined the ideal total hip replacement range of motion was 60 degrees of external rotation and 40 degrees of internal rotation [1] .

Whether this is enough for an impingement free ROM 
Table 3. Ranges of motion reported in the literature

\begin{tabular}{|l|l|l|l|l|l|l|l|}
\hline Movement & Flex & Ext & IR & ER & Abd & Add & Paper \\
\hline Range & $120^{\circ}$ & $30^{\circ}$ & $45^{\circ}$ & $45^{\circ}$ & $45^{\circ}$ & $35^{\circ}$ & Turley et al [4] \\
\hline & $113^{\circ}$ & $28^{\circ}$ & $45^{\circ}$ & $45^{\circ}$ & $48^{\circ}$ & $31^{\circ}$ & Boone et al [6] \\
\hline & $120^{\circ}$ & $9.5^{\circ}$ & $32^{\circ}$ & $33^{\circ}$ & $39^{\circ}$ & $30^{\circ}$ & Roaas et al [7] \\
\hline In 90' flex & & & $38^{\circ}$ & $40^{\circ}$ & & & $\begin{array}{l}\text { Kouyoumdjian } \\
\text { et al [5] }\end{array}$ \\
\hline
\end{tabular}

in-vivo is beyond the scope of the present study. This study does not include soft tissues in the model, which can potentially cause as well as prevent impingement by altering or limiting the ROM arc.

Of clinical relevance, an inferiorly placed elevated lip will increase jump distance postero-inferiorly. The relevance of this is in combined ROM, in particular flexion and internal rotation where the impingement is between the antero-superior acetabulum (or soft tissues) and anterior neck. This is a common direction of dislocation (as when sitting in a low chair or internally rotating whilst getting up from a seated position) and as such there may be some benefit in positioning of the lip in this location without the consequent loss of primary arc. For impingement on the inferiorly placed lip to occur, one would have to externally rotate $>140$ degrees (Figure 4a), which is well outside the physiological ranges for function.

With paucity of data in the literature regarding lip liner position that may improve hip stability, biomechanically and with soft tissue effects aside, our study shows that an inferiorly placed lip liner will allow increased hip abduction compared to a traditionally superiorly / postero-superiorly placed lip liner. Whilst abduction is generally a safe position unless in extreme range (as in performing a split) and therefore not of concern in the vast majority of hip replacement patients the value of increasing inferior jump distance may be in mixed abduction and flexion activities (riding a horse or a jetski) as demonstrated above. Further, range (allowance and restriction) and potential impingement [32].

The ROM results presented in the present study were mainly monoplanar, except the one combination movement tested of flexion with internal/external rotation. This simple model does not take into account complex movements of the hip joint that a patient may sometimes undertake in their daily living. However, accounting for the above, based on our results, an inferiorly placed lip is likely to be protective in particular mixed movement that are traditionally part of the 'hip precautions' i.e., avoidance of flexion/IR, and as such may have significant merit. Optimum implant position for that patient is still a prerequisite.

\section{Conclusion}

The findings of this study indicate that, provided optimum implant position for that patient, an inferiorly placed elevated lip liner, may provide additional stability with hip abduction and possibly in combined flexion/IR thus allowing patients a greater range of motion in those planes before dislocation can occur.
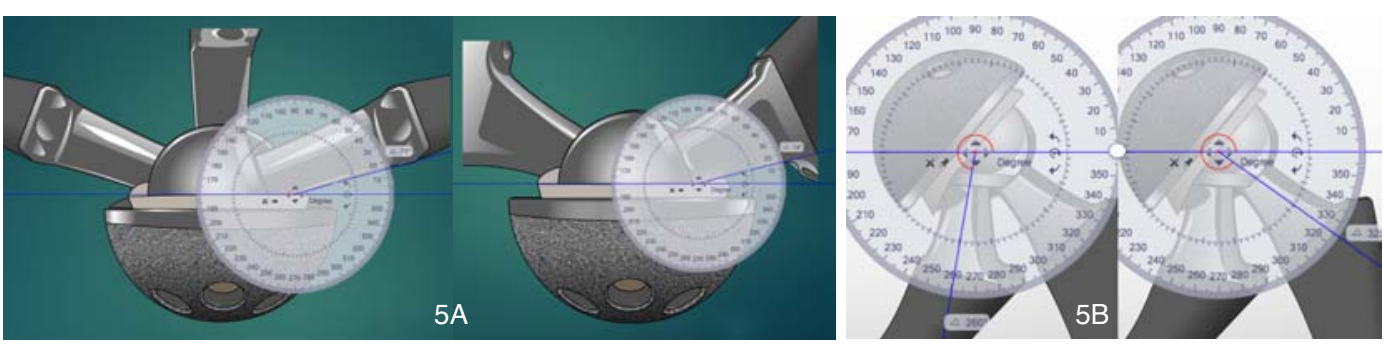

Figure 5a) Rotation and Abduction/ Adduction arc limited by implant design with no lip. b)Adduction range with inferior position of Lip liner c) $3 D$ representation - Flexion, Flexion / Adduction, Flexion/ Adduction/IR

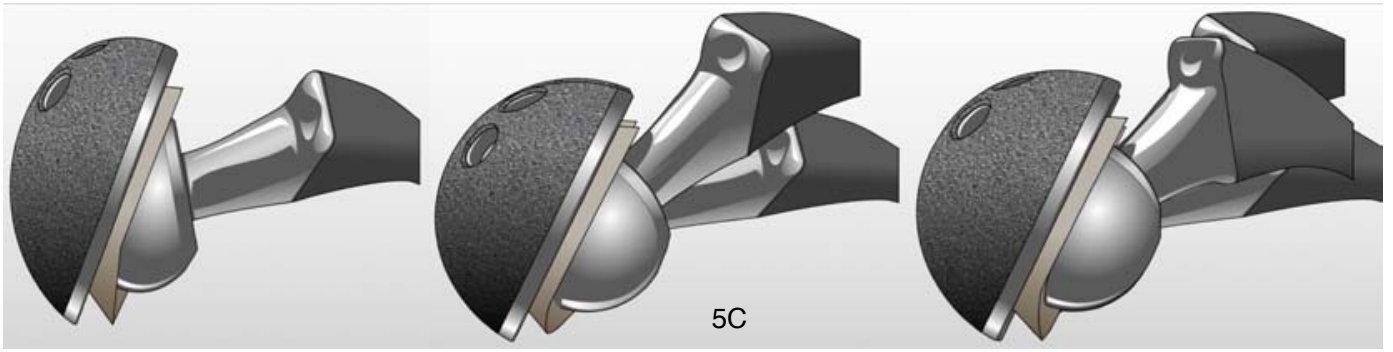




\section{References:}

1. Australian National Joint Replacement Registry. Annual Report 2015.

2. Qurashi et al, SuperPATH Minimally Invasive Total Hip Arthroplasty - An Australian Experience, JISRF Reconstructive Review, Vol 6, No.2,July 2016.

3. Matta $\mathbf{J}$ et al. Single-incision anterior approach for total hip arthroplasty on an orthopaedic table. Clin Orthopaedics and Related Research. 2005;441:115-124.

4. Turley GA et al. Evaluation of range of motion restriction within the hip joint. Med Biol Eng Comput. 2013;51:467-477.

5. Kouyoumdjian P, Coulomb R, Sanchez T, Asencio G. Clinical evaluation of hip joint rotation range of motion in adults. Orthop Traumatol Surg Res 2012;98:1723.

6. Boone DC, Asen SP. Normal range of motion of joints in male patients. J Bone Joint Surg Am 1979 Jul;61(5):756-9.

7. Roaas A, Andersson GB. Normal range of motion of the hip, knee and ankle joints in male subjects, 30-40 years of age. Acta Orthop Scand. 1982 Apr;53(2):205-8.

8. Insull PJ, Cobbett H, Frampton CM, Munro JT. The use of a lipped acetabular liner decreases the rate of revision for instability after total hip replacement. Bone Joint J 2014;96-B:884-8.

9. Cobb TK, Morrey BF, Ilstrup DM: The elevated-rim acetabular liner in total hip arthroplasty: relationship to postoperative dislocation. J Bone Joint Surg Am 78:80, 1996.

10. Brooks PJ. Dislocation following total hip replacement. Bone Joint J 2013;95-B, Supple A:67-9.

11. Krushell RJ, Burke DW, Harris WH. Elevated-rim acetabular components. Effect on range of motion and stability in total hip arthroplasty. J Arthroplasty. 1991;6Suppl:S53-8.

12. Charnley J: Low friction arthroplasty of the hip: theory and practice. Springer, New York, 1979.

13. Singh SP, Bhalodiya HP. Head size and dislocation rate in primary total hip arthroplasty. Indian J Orthop. 2013 Sep-Oct; 47(5):443-448.

14. Morrey BF. Instability after total hip arthroplasty. Orthop Clin North Am. 1992;23:237-48.

15. Crowninshield RD, Maloney WJ, Wentz DH, Humphrey SM, Blanchard CR. Biomechanics of large femoral heads: what they do and don't do. Clin Orthop Relat Res. 2004 Dec;(429):102-7

16. Scifert et al. Finte element analysis of a novel design approach to resisting total hip dislocation.

17. Shon WY et al. Impingement in Total Hip Arthroplasty: A study of retrieved acetabular components. J Arthroplasty 2005.

18. Sultan PG, Tan V, Lai M, Garino JP. Independent contribution of elevated-rim acetabular liner and femoral head size to the stability of total hip implants. J Arthroplasty. 2002 Apr;17(3):289-92.

19. Dargel J, Oppermann J, Bruggeman GP, Eysel P. Dislocation following total hip replacement. Dtsch Arztebl Int. 2014 Dec;111(51-52):884-890.

20. Yamaguchi M, Akisue T, Bauer TW et al. The spatial location of impingement in total hip arthroplasty. J Arthroplasty 2000;15:305.

21. Parr, W. C. H., Chatterjee, H. J., Soligo, C. (2012a). Calculating the axes of rotation for the subtalar and talocrural joints using $3 \mathrm{D}$ bone reconstructions. Journal of Biomechanics, 45, 1103-1107.

22. Parr, W. C. H., Soligo, C., Smaers, J., Chatterjee, H. J., Ruto, A., Cornish, L., \& Wroe, S. (2014). Three dimensional shape variation of talar surface morphology in hominoid primates. Journal of anatomy, 225(1), 42-59.

23. Parr, W. C. H., Wroe, S., Chamoli, U., Richards, H. S., McCurry, M. R., Clausen, P. D., \& McHenry, C. (2012b). Toward integration of geometric morphometrics and computational biomechanics: New methods for 3D virtual reconstruction and quantitative analysis of Finite Element Models. Journal of Theoretical Biology, 301, 1-14.

24. Parr, W.C.H., Chamoli, U., Jones, A., Walsh, W.R., Wroe, S., (2013). Finite Element micro-modelling of a human ankle bone reveals the importance of the trabecular network to mechanical performance: New methods for the generation and comparison of 3D models. Journal of Biomechanics, 46, 200-205.
25. Scheerlink T. Cup positioning in total hip arthroplasty. Acta Orthop. Belg. 2014;(80):336-347.

26. Lewinnek GE, Lewis JL, Tarr R, Compere CL, Zimmerman JR. Dislocations after total hip-replacement arthroplasties. J Bone Joint Surg Am. 1978;60:217-220.

27. Pierrepont J, Hawdon G, Miles BP, et al. Variation in functional pelvic tilt in patients undergoing total hip arthroplasty. Bone Joint J. 2017 Feb;99-B(2):184-191. Zahar A, Rastogi A, Kendoff D. Dislocation after total hip arthroplasty. Curr Rev Musculoskelet Med. 2013 Dec; 6(4):350-356.

28. Brown TD, Callaghan JJ. Impingement in total hip replacement: Mechanisms and consequences. Curr Orthop 2008. 22:376-391.

29. Tanino H, Harman MK, Banks SA, Hodge WA. Association between dislocation, impingement, and articular geometry in retrieved acetabular polyethylene cups. J Orthop Res 2007.25:1401-1407.

30. Usrey MM, Noble PC, Rudner LJ, Conditt MA, Birman MV, Santore RF, Mathis KB. Does neck/liner impingement increase wear of ultra-high-molecular-weight polyethylene liners? J Arthroplasty 2006. 21:65-71.

31. Cibulka MT, Strube MJ, Meier D, Selsor M, Wheatley C, Wilson NG et al. Symmetrical and asymmetrical hip rotation and its relationship to hip rotator muscle strength. Clin Biomech (Bristol, Avon) 2010;25:56-62 .

32. Widmer KH, Majewski M. The impact of the CCD-angle on range of motion and cup positioning in total hip arthroplasty. Clin Biomech (Bristol, Avon) 2005;20:723-8.

33. Bourne RB, Rorabeck CH. Soft tissue balancing: The hip. J Arthroplasty. 2002 Jun;17(4 Suppl 1):17-22

34. Longjohn D, Dorr LD. Soft tissue balance of the hip. J Arthroplasty. 1998 Jan;13(1):97-100.

\section{SUBMISSION HISTORY}

Submitted October10, 2017

Reviewed November 11, 2017

Revised November 19, 2017

Accepted December 14, 2017

Published December 31, 2017

\section{AUTHOR AFFILIATIONS}

1 Dr Suleman Qurashi

Department of Orthopaedic Surgery, Nepean Hospital, NSW, Australia

2 Dr William Parr, Dr William R Walsh

Surgical and Orthopaedic Research Laboratories (SORL), Prince of Wales Clinical

School, Prince of Wales Hospital, University of New South Wales (UNSW),

Randwick, NSW, 2031, Australia

3 Dr Bob Jang, Department of Orthopaedic Surgery, Canterbury Hospital, NSW, Australia

(Direct inquires to Bob Jang, jang.bob@gmail.com)

AUTHOR DISCLOSURES

The authors declare there are no disclosures regarding the publication of this paper.

COPYRIGHT \& OPEN ACCESS

C 2017 Qurashi, Parr, Jang, Walsh. All rights reserved.

Authors retain copyright and grant the journal right of first publication with the work Reconstructive Review is an open access publication and follows the Creative Commons Attribution-NonCommercial CC BY-NC. This license allows anyone to download works, build upon the material, and share them with others for non-commercial purposes as long as they credit the senior author, Reconstructive Review, and the Joint Implant Surgery \& Research Foundation (JISRF). An example credit would be: "Courtesy of (senior author's name), Reconstructive Review, JISRF, Chagrin Falls, Ohio". 